\title{
Prevalencia de infecciones hospitalarias en unidades de cuidados intensivos para adultos en Argentina
}

\author{
Guillermo R. Lossa, ${ }^{1}$ Roberto Giordano Lerena, ${ }^{1}$ Laura Elena Fernández, ${ }^{1}$ \\ Juana Vairetti, ${ }^{1}$ Carolina Díaz, ${ }^{1}$ Diego Arcidiácono ${ }^{1}$ y Norma Peralta ${ }^{1}$
}

Forma de citar Lossa GR, Giordano Lerena R, Fernández LE, Vairetti J, Díaz C, Arcidiácono D, et al. Prevalencia de infecciones nosocomiales en unidades de cuidados intensivos para adultos en Argentina. Rev Panam Salud Publica. 2008;24(5):324-30.

RESUMEN Objetivos. Presentar resultados consolidados de dos encuestas de prevalencia de infecciones nosocomiales, realizadas en el marco del Programa Nacional de Vigilancia de Infecciones Hospitalarias de Argentina.

Métodos. Se realizaron dos ediciones de la Encuesta Nacional de Prevalencia de Infecciones Hospitalarias de Argentina, en 2004 y 2005. De 68 hospitales, 53 notificaron datos de unidades de cuidados intensivos de adultos polivalente (26 en 2004 y 27 en 2005), con un total de 359 pacientes (158 en 2004 y 201 en 2005). La encuesta se diseñó como un estudio multicéntrico, observacional, transversal y descriptivo. El análisis de los datos se realizó utilizando distintos paquetes estadísticos, con variables y tiempos unificados y estandarizados para permitir la incorporación de los hospitales de ambos periodos.

Resultados. La prevalencia de pacientes con infección hospitalaria fue de $24 \%$. Sobre 127 episodios, el más frecuente fue la neumonía (43,3\%), que en $85 \%$ de los casos se asoció a asistencia respiratoria mecánica. En segundo lugar, se encontró la infección primaria de la sangre con $20,5 \%$; en $61 \%$ de los casos se la asoció a catéter central. Los pacientes expuestos a asistencia respiratoria mecánica presentaron mayor riesgo $(\mathrm{P}<0,001)$ de desarrollar neumonía; esto no se observó en pacientes con catéter central o urinario.

Conclusiones. La prevalencia de infección hospitalaria, la distribución por sitios primarios y la asociación a factores de riesgo, fueron similares a lo observado por otros autores de distintos países. Es fundamental conocer la problemática local y nacional antes de iniciar un programa de vigilancia. Estas encuestas, de carácter nacional y administradas por el Estado, son un factor de motivación, concienciación y capacitación.

Palabras clave Infecciones nosocomiales, cuidados intensivos, prevalencia, control de infecciones, Argentina.

1 Instituto Nacional de Epidemiología "Juan H Jara", Mar del Plata, Argentina. La correspondencia deberá enviarse al doctor Guillermo R. Lossa. Rodriguez Peña 4832, Mar del Plata 7600, Argentina. T: 54-22 3472-0077. Correos electrónicos: guillermo@ lossa.com.ar y grlossa@ecolan.com.
Las infecciones hospitalarias (IH) afectan de $4 \%$ a $13 \%$ de los pacientes que ingresan al nosocomio, y son más frecuentes en las unidades de cuida- dos intensivos. A mayor complejidad de los pacientes asisistidos en los hospitales mayor es el riesgo de adquirir una IH. En los pacientes quirúrgicos, 
el riesgo aumenta según el tipo de herida (limpia, limpia contaminada, contaminada o sucia).

Las IH generan un considerable incremento en la morbimortalidad, con el consiguiente aumento de costos en hospitalización (1-6). Se consideran un problema endemoepidémico, controlable, pero difícilmente erradicable; sin embargo, mediante programas eficaces se puede reducir notablemente su incidencia y disminuir drásticamente la morbilidad y los costos, optimizando la asignación limitada de recursos para la salud (7-11).

En Argentina, para esta última década sólo existen datos parciales de estudios presentados en forma aislada, acotada e intermitente. En la Encuesta Nacional de Prevalencia de Infecciones Hospitalarias de Argentina (ENPIHA) que se aplicó a los nosocomios que se incorporarían más tarde al proceso de vigilancia intensiva de las $\mathrm{IH}, 59 \%$ de éstos informaron no tener datos propios sobre porcentaje de IH (12).

El Programa Nacional de Vigilancia de Infecciones Hospitalarias de Argentina (VIHDA), del Ministerio de Salud de la Nación, tiene su sede operativa en el Instituto Nacional de Epidemiología "Dr. Juan H. Jara" (INE) de la ciudad de Mar del Plata, en Argentina, mismo que depende de la Administración Nacional de Laboratorios e Institutos de Salud (ANLIS) y que contó para su concreción con el apoyo del Programa de las Naciones Unidas para el Desarrollo (PNUD), en el Proyecto de Vigilancia de Infecciones de Argentina (VIGI+A).

El Programa VIHDA establece y coordina una red de hospitales en todo el país que permite, a partir de la información de cada institución, generar una gran base de datos representativa de la realidad nacional. De allí se obtienen indicadores inmediatos y actualizados de episodios de IH y se realiza, en forma continua, vigilancia epidemiológica. El software VIHDA es la plataforma informática diseñada específicamente para soportar la información y la gestión del Programa, en cuyo marco se realizaron dos ediciones de la
ENPIHA, en 2004 y 2005, con 68 nosocomios participantes, de los cuales 53 notificaron datos relativos a las unidades de cuidados intensivos de adultos polivalente (UCIA-POL).

\section{Objetivos de la Encuesta Nacional de Prevalencia de Infecciones Hospitalarias de Argentina}

Se buscó realizar un estudio de prevalencia de pacientes con $\mathrm{IH}$ en las UCIA-POL de los hospitales que se incorporaron voluntariamente al Programa VIHDA en los años 2004 y 2005. Los nosocomios que participaron fueron distintos en cada ocasión. De esta forma, se elaboró la ENPIHA, que permitió: a) conocer la situación basal de los hospitales de Argentina en relación con la prevalencia de episodios de IH en las UCIA-POL, evaluados bajo un mismo marco metodológico; b) describir características de los pacientes internados en dichas unidades, para determinar la población más vulnerable y los factores asociados con la adquisición de IH, y c) conocer las localizaciones más comunes de IH según tipo de UCIA-POL, los microorganismos más frecuentes, los antibióticos más utilizados y la resistencia hallada.

\section{MATERIALES Y MÉTODOS}

Se llevó a cabo un estudio multicéntrico, observacional, transversal y descriptivo, de conveniencia y no probabilístico. Se incluyeron hospitales con más de 50 camas, que poseyeran una UCIA-POL, con un comité de IH y una enfermera especializada en control de IH.

Los hospitales participaron voluntariamente, con consentimiento expreso de sus directores y como paso previo a la incorporación al sistema de vigilancia continua. Se diseñó un manual con planillas, instructivos, definiciones y criterios, y se capacitó a los profesionales participantes. Asimismo se elaboró un software ad hoc y se entregó un instructivo para su instalación y funcionamiento.
La ENPIHA se aplicó un solo día del mes de junio de 2004 y de 2005, en todos los hospitales, empleando metodología y pautas uniformes. Los integrantes de los comités de IH de cada nosocomio, previamente capacitados por profesionales del Programa VIHDA, se hicieron cargo de la captura de datos. Se incluyó a todos los pacientes internados por más de 24 horas en alguna UCIA-POL, sin otro criterio de exclusión. Las fuentes de información consultadas por el personal de los hospitales fueron la historia clínica del paciente, sus estudios radiológicos, los registros de enfermería y los informes del laboratorio de microbiología.

Se consideró como IH a toda infección adquirida durante la internación, que no estuviese presente o incubándose al momento de la admisión del paciente, de acuerdo con la definición de los Centros para el Control y la Prevención de las Enfermedades (CDC), del Departamento de Salud y Servicios Humanos de los Estados Unidos de América $(13,14)$. Se capturaron datos respecto de todos los pacientes internados en alguna UCIA-POL y episodios de IH en curso al momento de aplicar la encuesta. Posteriormente, los datos recopilados se enviaron al INE, donde se procedió a su análisis.

\section{Tratamiento de los datos}

En cada hospital se recolectó la información utilizando un software desarrollado expresamente por profesionales del Programa VIHDA, y que favoreció que la calidad y consistencia de los datos proporcionados por cada institución fueran validados al momento mismo de ser declarados. A continuación se notificó esa información al INE, donde se realizó el análisis de los datos de cada institución, a fin de garantizar su calidad. Finalizadas las correcciones, se emitieron los informes particulares que se entregaron a los respectivos comités de IH de los nosocomios participantes.

Finalmente, se consolidaron los datos en una sola base y se aplicaron procesos de cálculo de indicadores globales pre- 
definidos mediante otro software creado para tal fin por el Programa VIHDA.

\section{Variables consideradas}

En la ENPIHA se tomaron en cuenta los criterios y definiciones (13-15) para $\mathrm{IH}$; se consideraron los sitios primarios y específicos de acuerdo con el National Nosocomial Infection Surveillance (NNIS) y la Clasificación Internacional de Enfermedades (CIE 10) y Procedimientos (CIE 9).

Por otra parte, en la ENPIHA también se incluyeron factores de riesgo endógenos, esto es, propios del paciente al ingreso a la UCIA-POL, como tabaquismo, enfermedad pulmonar obstructiva crónica, obesidad, accidente cerebro vascular, coma, diabetes, insuficiencia renal, inmunodeficiencia, alcoholismo, neoplasia, insuficiencia hepática, desnutrición, úlcera de decúbito y dependencia a alguna droga. Asimismo, se consideraron factores de riesgo exógenos, es decir, procedimientos realizados en los pacientes, con especial énfasis en asistencia respiratoria mecánica, catéter central y catéter urinario. En lo que respecta a episodios de IH se registró su asociación o no a los factores de riesgo exógenos, diagnóstico microbiológico, resistencia y tratamiento antibiótico administrado.

Con respecto a la población y a la distribución de pacientes, se obtuvieron datos según: a) edad; b) sexo; c) estancia en el servicio; d) diagnóstico de ingreso al servicio según categoría CIE 10; e) infección al ingreso; f) prevalencia de IH por sitios primarios, su asociación a factores de riesgo exógenos y su relación con factores de riesgo endógenos; g) tratamientos: empírico no confirmado bacteriológicamente, rotado confirmado bacteriológicamente, empírico luego confirmado bacteriológicamente, rotado no confirmado bacteriológicamente y confirmado bacteriológicamente desde el inicio, y h) microorganismos: relación entre IH y diagnóstico microbiológico, distribución de las muestras, distribución de microorganismos por sitio primario y resistencia antibiótica.

\section{Análisis de los datos}

La información se analizó de acuerdo con el cálculo de medidas de tendencia central y análisis univariante. En aquellos indicadores que guardaron relación con mayor riesgo de desarrollar $\mathrm{IH}$, se calculó la razón de posibilidades (RP) con $P<0,05$ y con corrección de Yates (Yates) e intervalo de confianza de 95\% (IC95\%). Las variables cualitativas se analizaron con la prueba de $\chi^{2}$.

\section{Universo de trabajo}

En la ENPIHA se recabaron datos de 53 hospitales que poseían UCIA-POL ( 26 nosocomios en 2004 y 27 en 2005), pertenecientes a 21 provincias y a la Ciudad Autónoma de Buenos Aires (sobre un total de 23 provincias) de la República Argentina (cuadros 1 y 2). Del total de nosocomios, $40 \%$ notificaron entre 100 y 200 camas y $23 \%$ entre 200 y 300 camas. Por otra parte, $86 \%$ de los hospitales informaron un promedio de ocupación mayor a $60 \%$, y $39 \%$ uno mayor a $80 \%$.

En 2004 se registraron datos de 26 UCIA-POL que, en total, poseían 241 camas, con un promedio de $9,3 \%$ por hospital. El porcentaje ocupacional fue de $65,6 \%$, correspondiente a 158 pacientes hospitalizados. En 2005 se recopilaron datos de 27 UCIA-POL, con un total de 261 camas y un promedio de $9,7 \%$ por hospital. El porcentaje ocupacional fue de $76,4 \%$, co-

\section{CUADRO 1. Hospitales de Argentina con UCIA-POLa participantes en la ENPIHA ${ }^{b}$} 2004

\begin{tabular}{cccc}
\hline Institución $^{c}$ & $\begin{array}{c}\text { Camas } \\
\text { institución }\end{array}$ & $\begin{array}{c}\text { Camas } \\
\text { UCIA-POL }\end{array}$ & Pacientes \\
\hline 1 & 325 & 8 & 8 \\
2 & 300 & 10 & 10 \\
3 & 226 & 10 & 5 \\
4 & 300 & 15 & 8 \\
5 & 432 & 8 & 8 \\
6 & 235 & 12 & 5 \\
7 & 400 & 10 & 6 \\
8 & 240 & 8 & 8 \\
9 & 115 & 8 & 6 \\
10 & 87 & 8 & 4 \\
11 & 160 & 7 & 6 \\
12 & 387 & 6 & 6 \\
13 & 130 & 9 & 3 \\
14 & 266 & 7 & 6 \\
15 & 293 & 8 & 8 \\
16 & 306 & 12 & 5 \\
17 & 102 & 6 & 9 \\
18 & 168 & 16 & 5 \\
19 & 158 & SD & 6 \\
20 & 190 & 6 & 3 \\
21 & 151 & 7 & 6 \\
22 & 334 & 14 & 5 \\
23 & 403 & 5 & 3 \\
24 & 155 & 8 & 3 \\
25 & 157 & 7 & 158 \\
26 & 190 & 241 & \\
26 & 6210 & & 3 \\
\hline Fuenten & & 9 & 5 \\
\hline
\end{tabular}

Fuente: Encuesta de prevalencia en 26 unidades de cuidados intensivos de adultos (2004).

a Unidades de cuidados intensivos de adultos polivalente.

b Encuesta Nacional de Prevalencia de Infecciones Hospitalarias de Argentina.

c Los nombres de los hospitales son confidenciales; el software los numera automáticamente.

d Sin datos. 
CUADRO 2. Hospitales de Argentina con UCIA-POL a participantes en la ENPIHA 2005

\begin{tabular}{|c|c|c|c|}
\hline Institución ${ }^{c}$ & $\begin{array}{l}\text { Camas } \\
\text { institución }\end{array}$ & $\begin{array}{c}\text { Camas } \\
\text { UCIA-POL }\end{array}$ & Pacientes \\
\hline 27 & 300 & 14 & 8 \\
\hline 28 & 230 & 10 & 8 \\
\hline 29 & 330 & 12 & 9 \\
\hline 30 & 170 & 6 & 6 \\
\hline 31 & 220 & 8 & 6 \\
\hline 32 & 361 & 10 & 9 \\
\hline 33 & 200 & 8 & 5 \\
\hline 34 & 404 & 19 & 17 \\
\hline 35 & 175 & 5 & 5 \\
\hline 36 & 150 & 6 & 3 \\
\hline 37 & 174 & 8 & 6 \\
\hline 38 & 280 & 13 & 13 \\
\hline 39 & 135 & 5 & 5 \\
\hline 40 & 497 & 22 & 19 \\
\hline 41 & 84 & 7 & 5 \\
\hline 42 & 92 & 5 & 3 \\
\hline 43 & 120 & 12 & 0 \\
\hline 44 & 223 & 4 & 0 \\
\hline 45 & 206 & 16 & 12 \\
\hline 46 & 106 & 6 & 5 \\
\hline 47 & 82 & 3 & 2 \\
\hline 48 & 82 & 8 & 5 \\
\hline 49 & 345 & 7 & 6 \\
\hline 50 & 75 & 13 & 12 \\
\hline 51 & 184 & 13 & 12 \\
\hline 52 & 91 & 9 & 8 \\
\hline 53 & 358 & 12 & 12 \\
\hline 27 & 5674 & 261 & 201 \\
\hline
\end{tabular}

Fuente: Encuesta de prevalencia en 27 unidades de cuidados intensivos de adultos (2005).

a Unidades de cuidados intensivos para adultos polivalente.

b Encuesta Nacional de Prevalencia de Infecciones Hospitalarias de Argentina.

c Los nombres de los hospitales son confidenciales; el software los numera automáticamente.

rrespondiente a 201 pacientes hospitalizados. Lo anterior suma un total, en los dos años, de 53 UCIA-POL, con 502 camas y 359 pacientes observados, considerando los datos consolidados de ambos años para el presente trabajo. La estancia promedio en las UCIA-POL fue de 10 días/paciente. Del total de pacientes, $56 \%$ eran varones; la edad promedio fue de 53,5 años con una desviación estándar de \pm 20 años; $51,8 \%$ de los pacientes eran mayores de 60 años. ${ }^{2}$

\footnotetext{
2 Para mayor información, en el sitio en Internet www.vihda.gov.ar., en la sección "Publicaciones ENPIHA" podrá encontrarse un pormenorizado detalle de las encuestas de cada año, mismas que no se abordan aquí de manera discriminada por razones de espacio.
}

\section{RESULTADOS}

Los diagnósticos más frecuentes al ingreso fueron insuficiencia respiratoria aguda, neumonía, enfermedad cerebro vascular, traumatismo encéfalo craneal y sepsis. El diagnóstico de patología infecciosa al ingreso a la UCIA-POL correspondió a 46,5\% de los pacientes.

Los factores de riesgo endógenos más notificados fueron, en orden de importancia: tabaquismo, enfermedad pulmonar obstructiva crónica, coma, accidente cerebro vascular y obesidad. Los que se asociaron con mayor frecuencia a IH fueron alcoholismo y obesidad (cuadro 3).

La prevalencia de pacientes con episodios de IH fue de 24,2\% (IC95\%:
19,96-29). En 127 episodios de IH, se observó que el sitio más frecuente fue la neumonía $(43,3 \%)$ y que $85,4 \%$ de estos padecimientos se asociaron a asistencia respiratoria mecánica. De los episodios, $69 \%$ tuvieron confirmación bacteriológica y en $68,3 \%$ de éstos predominaron los bacilos Gram negativos. Los microorganismos más frecuentes fueron Acinetobacter sp (AC), Pseudomona aeruginosa (PAE) y Staphylococcus aureus (SAU).

Por otra parte, se notificó infección primaria de la sangre en $20,5 \%$ de los episodios de IH; de éstos, $66,7 \%$ tuvieron confirmación bacteriológica. Los microorganismos más prevalentes fueron SAU, AC y Klebsiella sp (KL); 61,5\% de las infecciones primarias de la sangre se asociaron a catéter central. Las infecciones del tracto urinario $(13,4 \%)$ ocuparon el tercer lugar; $64,7 \%$ se asociaron a catéter urinario y $87,5 \%$ de los episodios tuvieron confirmación bacteriológica. Los microorganismos más frecuentes fueron KL y AC. Desde el punto de vista microbiológico predominaron los bacilos Gram negativos $(68,7 \%)$, entre los que destacaron AC y KL. Entre los microorganismos Gram positivos, SAU fue el que se aisló con mayor frecuencia.

Dado el escaso número de aislamientos informados, los datos relativos a resistencia antibiótica podrían no reflejar, necesariamente, el verdadero perfil de los microorganismos. En el cuadro 4 se presentan las resistencias más frecuentemente notificadas. Los antibióticos de mayor uso, en casos tanto de aislamiento de microorganismos como sin aislamiento, fueron Imipenem, Vancomicina, Amikacina y Ciprofloxacina.

Los pacientes que estuvieron expuestos a asistencia respiratoria mecánica presentaron RP = 7; IC95\% 3-16,79 y $\chi^{2}$ Yates de $28,34(P<0,01)$ para desarrollar neumonía asociada a dicha asistencia. No se verificó riesgo significativamente mayor para desarrollar infecciones primarias de la sangre asociadas a catéter central $(P=0,53)$ o infecciones del tracto urinario asociadas a catéter urinario $(P=0,93)$. En el cuadro 5 se ofrece un resumen de los hallazgos más importantes del trabajo. 
CUADRO 3. Relación entre infección hospitalaria y factores de riesgo endógenos más frecuentes. Hospitales de Argentina, 2004 y 2005

\begin{tabular}{|c|c|c|}
\hline FREnd $^{a}$ & $\begin{array}{l}\text { Episodios de } \mathrm{IH}^{\mathrm{b}} / \text { No. de pacientes } \\
\text { con FREnd }\end{array}$ & $\begin{array}{c}\text { Prevalencia de } \mathrm{IH} \text { según FREnd } \\
\%\end{array}$ \\
\hline Tabaquismo & 22 / 82 & 26,8 \\
\hline EPOCC & $22 / 67$ & 32,8 \\
\hline Coma & 22 / 64 & 34,4 \\
\hline $\mathrm{ACV}^{\mathrm{d}}$ & $14 / 53$ & 26,4 \\
\hline Obesidad & $17 / 44$ & 38,6 \\
\hline Alcoholismo & $14 / 30$ & 46,7 \\
\hline
\end{tabular}

Fuente: Encuesta de prevalencia en 53 unidades de cuidados intensivos de adultos (Argentina, 2004 y 2005).

a Factor de riesgo endógeno.

${ }^{b}$ Infección hospitalaria.

c Enfermedad pulmonar obstructiva crónica.

d Accidente cerebro-vascular.

CUADRO 4. Resistencias bacterianas más frecuentemente halladas en infecciones hospitalarias. Argentina, 2004 y 2005

\begin{tabular}{|c|c|c|c|c|c|c|c|c|}
\hline \multirow{2}{*}{$\begin{array}{c}\text { Micro- } \\
\text { organismos }\end{array}$} & \multicolumn{8}{|c|}{ Antibióticos } \\
\hline & $\mathrm{AMK}^{\mathrm{a}}$ & $C A Z^{b}$ & $\mathrm{CIP}^{\mathrm{C}}$ & GEN $^{d}$ & MEM $^{\mathrm{e}}$ & $\mathrm{IPM}^{\dagger}$ & VANg & $O X A^{h}$ \\
\hline$A C^{i}$ & $\begin{array}{c}8 / 14 \\
(57 \%)\end{array}$ & $\begin{array}{c}15 / 15 \\
(100 \%)\end{array}$ & $\begin{array}{l}14 / 15 \\
(93 \%)\end{array}$ & $\begin{array}{c}16 / 16 \\
(100 \%)\end{array}$ & $\begin{array}{l}1 / 13 \\
(8 \%)\end{array}$ & $\begin{array}{c}3 / 16 \\
(19 \%)\end{array}$ & & \\
\hline $\mathrm{KL}^{\mathrm{j}}$ & $\begin{array}{c}9 / 14 \\
(64 \%)\end{array}$ & $\begin{array}{l}11 / 14 \\
(80 \%)\end{array}$ & $\begin{array}{c}14 / 16 \\
(87,5 \%)\end{array}$ & $\begin{array}{l}12 / 14 \\
(86 \%)\end{array}$ & $\begin{array}{l}0 / 14 \\
(0 \%)\end{array}$ & $\begin{array}{l}1 / 14 \\
(7 \%)\end{array}$ & & \\
\hline$P A E^{k}$ & $\begin{array}{c}3 / 12 \\
(25 \%)\end{array}$ & $\begin{array}{c}2 / 16 \\
(13 \%)\end{array}$ & $\begin{array}{c}3 / 13 \\
(23 \%)\end{array}$ & $\begin{array}{c}5 / 13 \\
(38,5 \%)\end{array}$ & $\begin{array}{c}1 / 9 \\
(11 \%)\end{array}$ & $\begin{array}{c}2 / 10 \\
(20 \%)\end{array}$ & & \\
\hline SAU' & & & $\begin{array}{l}10 / 16 \\
(62 \%)\end{array}$ & & & & $\begin{array}{l}0 / 20 \\
(0 \%)\end{array}$ & $\begin{array}{l}14 / 22 \\
(64 \%)\end{array}$ \\
\hline $\mathrm{SCN}^{\mathrm{m}}$ & & & $\begin{array}{c}3 / 6 \\
(50 \%)\end{array}$ & & & & $\begin{array}{l}0 / 8 \\
(0 \%)\end{array}$ & $\begin{array}{c}4 / 8 \\
(50 \%)\end{array}$ \\
\hline
\end{tabular}

Fuente: Encuesta de prevalencia en 53 unidades de cuidados intensivos de adultos (Argentina, 2004 y 2005).

a AMK: Amikacina. $\quad$ h OXA: Oxacilina.

b CAZ: Ceftazidima. I AC: Acinetobacter sp.

c CIP: Ciprofloxacina. I KL: Klebsiella sp.

d GEN: Gentamicina. $\quad$ k PAE: Pseudomona aeruginosa.

e MEM: Meropenem. I SAU: Staphylococcus aureus.

f IPM: Imipenem. $\quad$ m SCN: Staphylococcus coagulasa negativo.

9 VAN: Vancomicina.

\section{DISCUSIÓN}

A pesar de que los estudios de prevalencia presentan el inconveniente de la inestabilidad de los datos (toda vez que se realizan en un momento puntual en las UCIA-POL consideradas), también permiten obtener una visión general del problema, en forma rápida y económica. De igual manera, sirven para establecer el diagnóstico de situación inicial antes de la realización de estudios intensificados de incidencia en las áreas más críticas, así como para evaluar periódicamente los avances logrados a través de la vigilancia intensificada y las medidas de control establecidas al respecto.

En este trabajo se notificó una estancia promedio de 10 días/paciente, valor que se encuentra dentro del rango descrito por otros autores en investigaciones análogas en Europa (3, 16). La prevalencia de episodios de IH fue de $24,2 \%$, similar a lo publicado en algunos estudios multicéntricos $(2,3$, 17). La IH más frecuente fue neumonía y $85,4 \%$ de los casos se asociaron a asistencia respiratoria mecánica, lo cual es acorde con lo publicado por distintos autores (1-17). En segundo término estuvieron las infecciones primarias de la sangre, asociadas a catéter central en $61,5 \%$ de los casos $(2,3,18)$. Llama la atención que las neumonías presenten mayor cantidad de episodios con diagnóstico microbiológico que las infecciones primarias de la sangre, lo cual no se observó en otros informes (2). Lo anterior podría deberse al incremento de episodios de infecciones primarias de la sangre con diagnóstico clínico y a que muchos pacientes que desarrollaron ese tipo de infecciones se hallaban tratados previamente con antibióticos por otro episodio anterior (18). Predominaron los Gram negativos, en concordancia con lo publicado por otros estudios relacionados $(2,4)$; los más frecuentemente aislados fueron AC y KL; entre los Gram positivos destacó SAU. Cabe señalar que hubo datos sobre resistencia antibiótica que no fueron completados por la totalidad de las instituciones y el número final resultó ser muy bajo para hacer cálculos estadísticamente significativos y establecer un perfil de resistencia. No obstante, algunas de las cifras halladas coinciden con otros trabajos $(4,16)$. Al considerar todos los episodios de IH, los antibióticos más utilizados fueron Imipenem, Vancomicina, Amikacina y Ciprofloxacina. La exposición a asistencia respiratoria mecánica constituyó un riesgo estadísticamente significativo $(P<0,001)$ para desarrollar neumonía, lo cual coincide con lo observado por otros autores $(1,2,16,19)$. No obstante, en lo relativo al uso del catéter central y del urinario, existen diferencias con lo establecido en algunos estudios de vigilancia $(3,18)$. Cabe destacar la importancia de haber utilizado datos validados para realizar comparaciones entre hospitales y obtener indicadores veraces, cuya necesidad demostraron Stover y Jarvis en un estudio multicéntrico realizado en 50 hospitales de Inglaterra (20).

Por otro lado, según lo manifiesta el clásico estudio $\operatorname{SENIC~}(7,9,21)$ "la sola implementación de programas simples para medición y control de IH, hace posible reducir la tasa de $\mathrm{IH}$ in- 
CUADRO 5. Resumen de los resultados de la encuesta de prevalencia en 53 unidades de cuidados intensivos de adultos, Argentina, 2004 y 2005

\begin{tabular}{ll}
\hline \multicolumn{1}{c}{ Característica } & Resultado \\
\hline No. de pacientes & 359 \\
Características de la población & \\
$\quad$ Femenino & $44 \%$ \\
$\quad$ Masculino & $56 \%$ \\
$\quad>60$ años & $51,8 \%$ \\
Infección al ingreso & $46,5 \%$ \\
Prevalencia de infección hospitalaria & $24,2 \%($ IC $95 \%$ 19,9-29) \\
& \\
Sitio primario más frecuente (y factor exógeno asociado) & $43,3 \%(85,4 \%)$ \\
$\quad$ Neumonía (asociada a asistencia respiratoria mecánica) & $20,5 \%(61,5 \%)$ \\
$\quad$ Infección primaria de la sangre (asociada a catéter central) & $13,4 \%(64,7 \%)$ \\
$\quad$ Infección del tracto urinario (asociada a catéter urinario) & \\
$\quad$ Sitio primario más frecuente con diagnóstico microbiológico positivo & $68 \%$ \\
$\quad$ Neumonía & $66,7 \%$ \\
$\quad$ Infección primaria de la sangre & $87,5 \%$ \\
$\quad$ Infección del tracto urinario & \\
\hline
\end{tabular}

cluso hasta en un 32\%". Por su parte, Wilson y colaboradores (22) demostraron, en el caso de 113 hospitales en Inglaterra, que el mero hecho de participar en un programa de vigilancia produce un efecto inmediato entre el personal del hospital, ya que aumenta la motivación para el intercambio de información y la revisión de prácticas y conductas preestablecidas. Asimismo se observó que, entre dicho personal, se genera la necesidad de profundizar la formación en temas epidemiológicos.

En una encuesta preliminar (12) sobre los hospitales que participaron en la ENPIHA, 59\% de ellos notificaron carecer de datos propios sobre porcentaje de IH. Luego de esta encuesta y a partir de su continuidad en el Programa VIHDA, los mismos nosocomios conformaron y

Ruano C, Maldonado J, Salazar R. Frecuencia de infección nosocomial en terapia intensiva: datos del proyecto PIN-FCM. Rev Cubana Hig Epidemiol. 2004;42(1):5-9.

2. Sociedad Española de Medicina Preventiva, Salud Pública e Higiene. Grupo de Trabajo EPINE (Encuesta de Prevalencia de las Infecciones Nosocomiales en España). Evolución afianzaron sus respectivos comités de infecciones y continúan manteniendo datos habituales sobre IH. Esto también ha motivado la revisión y actualización de conductas preestablecidas, por ejemplo, esquemas antibióticos empíricos (23) y profilaxis prequirúrgica, como así también la normalización para el manejo de procedimientos invasivos $(8,19,24,25)$.

Es necesario promover políticas, en todos los niveles, que fortalezcan los sistemas de vigilancia efectiva. Esto es fundamental para conocer la problemática local y nacional y obtener indicadores propios, con el objeto de instaurar las medidas pertinentes. Además, en los hospitales de Argentina es una prioridad desarrollar y mantener una cultura de cooperación

\section{REFERENCIAS}

de la prevalencia de las infecciones nosocomiales en los hospitales españoles en 10 años. [Sitio en Internet] Hallado en: http://www. mpsp.org/mpsp/epine. Acceso el 30 de marzo 2001.

3. Llanos-Mendez A, Díaz-Molina C, Barranco Quintana J, García OV. Factores que influyen sobre la aparición de infecciones hospitalarias y trabajo multidisciplinario que inspire a los miembros del equipo a sentirse responsables por los resultados, a fin de disminuir la incidencia de $\mathrm{IH}$ $(7,9-11)$ y mejorar la calidad de la atención médica.

Este tipo de encuestas, de carácter nacional, y administradas por el Estado, son un factor de motivación, concienciación y capacitación. Constituyen, incluso, una invitación a la introspección, para que cada persona en el hospital -y este último como un todo-, se vea a sí misma y se pregunte si está haciendo las cosas bien, o si podría hacerlas mejor dentro de su contexto. Por otra parte, este tipo de estudios sienta las bases y establece conceptos, pautas, rutinas e indicadores que luego le permiten al hospital acceder más fácilmente a un programa de vigilancia intensificada de las $\mathrm{IH}$, como el que la República Argentina ha puesto en marcha desde 2004. Finalmente, se puede afirmar que éste es un ejemplo claro del uso de la "información para la acción", en aras de mejorar la calidad de la atención médica y disminuir la morbimortalidad por IH.

Agradecimientos. Este trabajo fue elaborado por el equipo ejecutivo del Programa VIHDA a partir de la información de los hospitales participantes que, más allá de completar la ENPIHA, estuvieron siempre a disposición del Programa para aclarar dudas, ajustar datos y brindar la interpretación clínica correspondiente a cada caso. Se agradece a todos ellos por la confianza depositada y la disponibilidad para contribuir al trabajo que aquí se presenta. Así mismo, se agradece la colaboración de todos los profesionales que, desinteresadamente, participaron en el mismo. 
llado en: www.who.int/entity/medicines/ publications/policyperspectives/ppm_10_sp. pdf Acceso el 25 de abril 2005.

6. Cosano A, Martínez-Gonzalez M, MedinaCuadros M, Martínez-Gallego S. Relationship between hospital infection and long-term mortality in general surgery: a prospective follow-up study. J Hosp Inf. 2002;52(2):122-9.

7. Morales Perez C, Guanche Garcell H, Núñez Labrador L, Fresneda Septiembre G. Sensibilidad de los sistemas de vigilancia de las infecciones nosocomiales. Rev Cub Enf. 2004; 20(2):1.

8. Luna CM, Monteverde A, Rodríguez A, Apezteguía C, Zabert G, Ilutovich S, et al. Clinical guidelines for the treatment of nosocomial pneumonia in Latin America: an interdisciplinary consensus document. Arch Bronconeumol. 2005;41:439-56.

9. Haley RW, Culver DH, White JW, Emori TG, Munn VP, Hooton TM. The efficacy of infection surveillance and control programs in preventing nosocomial infections in US hospitals. Am J Epidemiol. 1985;121(2):182-205.

10. Nguyen GT, Proctor SE, Jarvis WR. Status of infection surveillance and control programs in the United States, 1992-1996. Association for Professionals in Infection Control and Epidemiology, Inc. Am J Infect Control. 2000; 28(6):392-400.

11. Vegni FE, Panceri ML, Biffi E. Three scenarios of clinical claim reimbursement for nosocomial infection: the good, the bad, and the ugly. J Hosp Inf. 2004; 56(2):150-5.
12. Programa Nacional de Vigilancia de Infecciones Hospitalarias de Argentina. Informe Final Línea de Base Consolidado 2004 e Informe Final Línea de Base 2005. [Sitio en Internet] Hallado en: http://www.vihda.gov.ar/enpiha. Acceso el 13 de enero 2006.

13. Ganer JS, Jarvis WR, Emori TG, Horan TC, Hughes JM. CDC definitions of nosocomial infections. Am J Infect Control. 1998;16: $128-40$.

14. US Public Health Service, Centers for Disease Control and Prevention. National Nosocomial Infections Surveillance (NNIS) System Report, data summary from January 1992 through June 2004, issued October 2004. Am J Infect Control. 2004,32:470-85.

15. Gaynes R, Richards C, Edwards J, Grace Emori T, Horan T. Feeding back surveillance data to prevent hospital-acquired infections. Emerg Infect Dis. 2001; 7(2):295-8.

16. Urli T, Perone G, Acquarolo S. Surveillance of infections acquired in intensive care: usefulness in clinical practice. J Hosp Inf. 2002;52(2): 130-5.

17. Eggiman P, Pittet D. Infection control in the ICU. Chest 2001;120:2059-93.

18. Hugonnet S, Sax H, Eggiman P, Chevrolet JC, Pittet D. Nosocomial bloodstream infection and clinical sepsis. Emerg Infect Dis. 2004; 10(1)76-81.

19. Maraví-Poma E, Martínez Segura JM, Izura J, Gutierrez A, Tihista JA. Vigilancia y control de la neumonía asociada a ventilación mecánica. An Sist Sanit Navar. 2006; 23(2):143-60.
20. Stover BH, Shulman ST, Bratcher DF, Brady MT, Levine GL, Jarvis WR, et al. Nosocomial infection rates in US children's hospitals' neonatal and pediatric intensive care units. Am J Infect Control. 2001;29(3):152-7.

21. Department of Health and Human Services, Centres for Disease Control and Prevention. Public health focus: surveillance, prevention and control of nosocomial infections. MMWR. 1992;41:783-7.

22. Wilson JA, Ward VP, Coello R, Charlett A. A user evaluation of the Nosocomial Infection National Surveillance System: surgical site infection module. J Hosp Inf. 2002;52(2):114-21.

23. Allegranzi B, Luzzati R, Luzzani A, Girardini F. Impact of antibiotic changes in empirical therapy on antimicrobial resistance in intensive care unit-acquired infections. J Hosp Inf. 2002;56(2):136-40.

24. Uriz J, Salvador B, Gutierrez A, Petit A, Anguiano P, Sampériz A. Vigilancia y control de las bacteriemias asociadas a dispositivos vasculares. An Sist Sanit Navar. 2006;23(2): 161-8.

25. Ministerio de Salud de la República Argentina. Programa de Vigilancia de la Salud y Control de Enfermedades. Guías para la prevención y control de las infecciones nosocomiales. [Sitio en Internet] Hallado en: http:// www.vihda.gov.ar/publicaciones y trabajos. Acceso el 30 de enero 2006.

Manuscrito recibido el 5 de enero de 2007. Aceptado para publicación, tras revisión, el 19 de mayo de 2008.

ABSTRACT Objectives. To present consolidated results from two surveys of nosocomial infection incidence, within the framework of Argentina's National Surveillance of Hospital Infections Program.

Prevalence of hospital infections in adult intensive Methods. Two editions of the National Survey on the Incidence of Hospital Infections in Argentina were used, those of 2004 and 2005. Of the 68 hospitals, 53 reported data from adult intensive care units (in 2004, 26 units; in 2005, 27), for a total of 359 patients (in 2004, 158 patients; in 2005, 201). The survey was designed as a multicenter, observational, cross-sectional, and descriptive study. Data analysis was performed with several statistics programs, combining and standardizing variables and both time periods in order to include all the hospitals from the two surveys.

Results. The prevalence of hospital infection among patients was $24 \%$. Of the 127 illnesses, the most frequently occurring was pneumonia $(43.3 \%)$, which in $85 \%$ of the cases was associated with use of a respirator. Primary bloodstream infections took second place, at $20.5 \%$, with $61 \%$ of these cases being associated with a central catheter. Patients given respiratory therapy were at greatest risk $(P<0.001)$ of developing pneumonia; this was not observed among patients with central or urinary catheters.

Conclusions. The prevalence of hospital infections, the distribution of primary infection sites, and the associations with risk factors were similar to those observed by other authors in several countries. It is important to understand the local and national issues prior to initiating a surveillance program. Surveys such as these, with a national scope and administered by the State, are one way of motivating, raising awareness, and building capacity.

Key words Cross infection, intensive care, prevalence, infection control, Argentina. 